\title{
Evaluation of Uncertainties Related to the Near Field Model of Geological Repository for Spent Nuclear Fuel
}

\author{
Dana Barátová, Vladimír Nečas \\ Institute of Nuclear and Physical Engineering,Slovak University of Technology in Bratislava, Slovakia \\ Email: dana_baratova@stuba.sk, vladimir.necas@stuba.sk
}

Received 13 August 2015; accepted 15 October 2015; published 22 October 2015

\begin{abstract}
In Slovakia, a direct disposal of spent nuclear fuel in a deep geological repository within the country after a certain period of interim storage is a preferred option. This paper briefly describes near field model of radionuclide migration developed in GoldSim simulation code environment and analyses the calculated results on time-dependent release rates of safety relevant radionuclides. Given the fact that GoldSimalso enables to perform probabilistic simulations using the Monte Carlo method, a probabilistic approach was chosen to assess the influence of selected near field parameter uncertainties related to radionuclide migration on the radionuclide release rates from the bentonite buffer to the surrounding host rock. Based on the results, release rates of nuclides which exceed their solubility limits are effectively lowered and many of nuclides are significantly sorbed on the buffer material. It can be seen that the variance of the total release rate in the case of solubility uncertainty is almost two orders of magnitude within a long period of time.
\end{abstract}

\section{Keywords}

Geological Disposal, Near Field Model, GoldSim, Uncertainty, Probabilistic Simulation

\section{Introduction}

Deep geological repository as an alternative for spent fuel management is expected to provide sufficient protection of human health and the environment for at least several thousand years. Likelihood of the potential radiological impacts is higher in consideration of processes such as a gradual degradation of protective technical barriers or an intrusion caused by human activities. To ensure protection of human health and the environment, it is necessary to design a complex system of protective measures as well as to perform a comprehensive safety analysis in every period of the repository lifetime.

Spent fuel assemblies discharged from the VVER nuclear reactors in Slovakia are cooled several years in storage basins in the vicinity of the reactor [1]. After this period, the spent fuel is transported from the nuclear power plant to the interim spent nuclear fuel storage facility in Jaslovské Bohunice [2]. The spent fuel assemblies are in this facility stored in the pools filled with water (so called wet type of storage) [1].

How to cite this paper: Barátová, D. and Nečas, V. (2015) Evaluation of Uncertainties Related to the Near Field Model of Geological Repository for Spent Nuclear Fuel. World Journal of Engineering and Technology, 3, 163-169.

http://dx.doi.org/10.4236/wjet.2015.33C024 
Nowadays, as for the final stage of the spent fuel management, a direct disposal of spent nuclear fuel in a deep geological repository within the country after a certain period of interim storage is the preferred option. In Slovakia, the site selection process of the host rock environment suitable for geological disposal is still running but until now there has not been selected the final locality for a geological disposal facility. Due to this fact, the assessments of long-term safety were performed only for a hypothetical geological repository located in the crystalline or sedimentary rocks.

\section{Reference case}

The reference case is based on the reference scenario which represents the expected evolution of a disposal system with respect to processes and events which will occur with high probability within the gradual process of repository evolution. Radionuclides are transported by groundwater as a groundwater scenario is the most likely way how the biosphere will be contaminated by radionuclides from the spent nuclear fuel.

\subsection{Near Field Model}

Spent fuel is a complex and heterogeneous system and therefore was within the model conceptually divided into the structural material, $\mathrm{UO}_{2}$ matrix and instant release fraction (IRF). IRF is a fraction of inventory which is (after water contact) released rapidly, in the term of long-term safety instantaneously [3]. According the international safety assessments and certain assumptions as high solubility limits, relatively long half-lives, poor sorption on bentonite buffer, significant IRF values, 36 radionuclides were identified as relevant for the safety analysis. Inventory of ${ }^{14} \mathrm{C}$ was divided between the structural material and $\mathrm{UO}_{2}$ matrix. It is considered that ${ }^{14} \mathrm{C}$ originated from the structural material is presented in organic compounds and ${ }^{14} \mathrm{C}$ originated from $\mathrm{UO}_{2}$ matrix is a part of inorganic compounds [4].

The geological repository design is based on the principle of multi-barrier concept - combination of engineering barriers and a suitable geological formation. One of the barriers is the very form of spent fuel which dissolution rate in the contact with the groundwater is very low. Another barrier is represented by disposal container which parameters and physical and chemical properties are based on [4] [5]. The disposal capacity of one container is 7 fuel assemblies [5]. Disposal container is surrounded by a bentonite buffer with the wall thickness of $300 \mathrm{~mm}$. It provides a self-sealing, low permeable barrier because of its swelling potential.

Conceptual model of radionuclide migration through the engineered barrier system (EBS) takes into account the following main assumptions:

- Disposal container fails after 1000 years,

- Spent nuclear fuel is divided into the $\mathrm{UO}_{2}$ matrix and structural materials,

- Certain inventory fraction is after water-contact released instantaneously, long-term release occurs congruently with the dissolution of individual materials,

- Concentration of radionuclides is in the void volume of disposal container and in the bentonite buffer limited by elemental solubility,

- It is considered that the bentonite buffer is fully water-saturated that is why nuclides migrate through the buffer by radial diffusion,

- Contaminants are linearly sorbed on the material of bentonite buffer.

Modelling was carried out by using the simulation software GoldSim which RT module allows users to dynamically model mass transport within the complex system of engineering and natural barriers [6]. Mass transport is calculated based on the mass balance equation whereby it is also possible to simulate a radioactive decay and in growth of individual radionuclides. Equation (1) represents the basic mass balance equation for the cell i [6]:

$$
m_{i s}^{\prime}=-m_{i s} \lambda_{s}+\sum_{p=1}^{N P_{s}} m_{i p} \lambda_{p} f_{p s} R_{s p} \frac{A_{s}}{A_{p}}+\sum_{c=1}^{N F_{i}} f_{c s}+S_{i s}(\mathrm{~kg} / \mathrm{s})
$$

where:

$$
\begin{aligned}
& m_{i s}^{\prime} \text {-rate of increase of mass of species } \mathrm{s} \text { in cell } \mathrm{i}(\mathrm{kg} / \mathrm{s}) \text {, } \\
& m_{i s} \text {-mass of species s in cell } \mathrm{i}(\mathrm{kg}), \\
& \lambda_{\mathrm{s}} \text {-decay rate for species } \mathrm{s}\left(\mathrm{s}^{-1}\right),
\end{aligned}
$$


$N P_{s}$-number of direct parents for species $\mathrm{s}$,

$f_{p s}$-fraction of parent $\mathrm{p}$ which decays into species $\mathrm{s}$,

$R_{s p}$-stoichiometric ratio of moles of species s produced per mole of species p decayed,

$A_{s}$-molecular (or atomic) weight of species s $(\mathrm{kg} / \mathrm{mol})$,

$A_{p}$-molecular (or atomic) weight of species p ( $\left.\mathrm{kg} / \mathrm{mol}\right)$,

$N F_{i}$-number of mass flux links from/to cell $\mathrm{i}$,

$f_{c s}$-influx rate of species s (into cell i) through mass flux link c (kg/s),

$S_{\text {is }}$-rate of direct input of species s to cell i from "external” sources $(\mathrm{kg} / \mathrm{s})$.

\subsection{Reference Case Results}

Release rates were calculated based on the near field model where a source term is represented by 7 fuel assemblies (one disposal container) with the initial average enrichment of $4.87 \%$ of ${ }^{235} \mathrm{U}$ and burnup $60 \mathrm{MWd} / \mathrm{kgU}$. Figure 1 and Figure 2 illustrate the time-dependent release rates of radionuclides from the EBS which represent the largest contribution to the total release rate.

Based on the results of time-dependent release rates the following conclusions can be done:

- ${ }^{227} \mathrm{Ac},{ }^{210} \mathrm{~Pb},{ }^{226} \mathrm{Ra}$ are radionuclides with large inventories and low solubility limits. This results in a pseudo-steady-state release rates for a significant time,

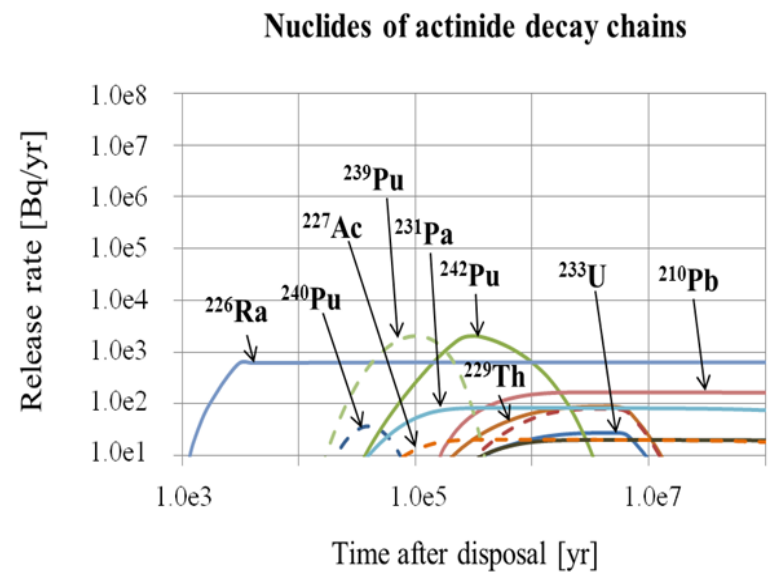

Figure 1. Release rates of nuclides of actinide decay chains from the EBS per one disposal container.

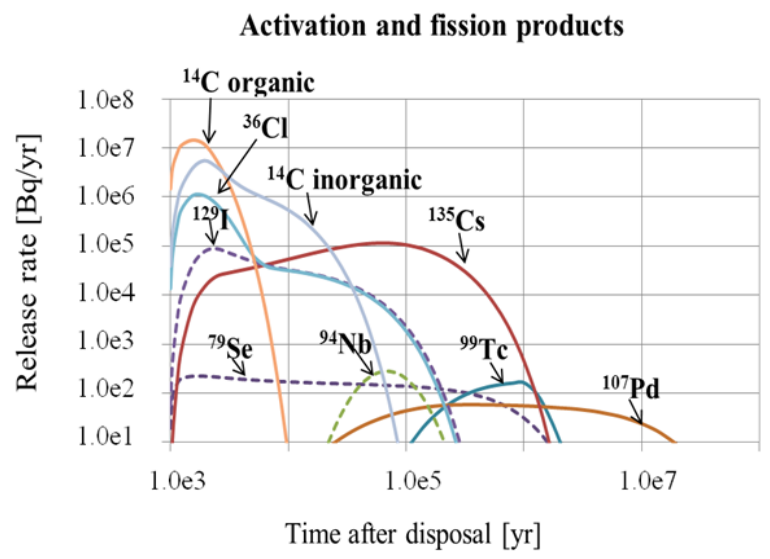

Figure 2. Release rates of nuclides of activation and fission products from the EBS per one disposal container. 
- Release rates of radionuclides with significant distribution coefficients are very small,

- Activation and some of the fission products $\left({ }^{135} \mathrm{Cs},{ }^{129} \mathrm{I}\right)$ represent the largest contribution to the total release rate in the early years of safety analysis,

- Due to the poor retentive properties, activation and some of the fission products $\left({ }^{135} \mathrm{Cs},{ }^{79} \mathrm{Se}\right)$ relatively quickly migrate through the buffer what can be seen from their time-dependent release rate curves.

\section{Probabilistic Modelling of Selected Parameter}

Due to the lack of research and development works in the field of geological disposal in Slovakia, near field model was developed by using the international research and development achievements (Czech Republic-disposal container and geometry of bentonite buffer, Switzerland-bentonite buffer properties). It is also known that a long-term safety cannot be clearly evaluated because of the incompleteness of our knowledge about a given system and also because of the variability in the space and time.

To evaluate the impact of uncertainties related to the solubility and sorption in the near field on the release of radionuclides from the EBS, the sensitivity analysis of parameters corresponding to these processes was performed. Because GoldSim also offers an option of probabilistic simulation using the Monte Carlo method, aprobabilistic approach was chosen to perform a sensitivity analysis.

In the probabilistic assessment, parameter uncertainties are quantified in terms of probability density functions. Solubility limits and distribution coefficients were in the probabilistic calculations specified as stochastic input parameters using the discrete distribution which is described mathematically by probability mass function, rather than probability density function. Probability mass functions specify actual probabilities for given values, rather than probability densities

\subsection{Selected Parameters}

\subsubsection{Distribution Coefficient $K_{d}$}

Sorption is the process in which dissolved contaminants partition from the ground water and adhere to the particles of e.g. bentonite buffer. Sorption is a reversible reaction which means that at a given solute concentration, some portion of the solute is partitioning to the bentonite material and some portion is also desorbing and reentering the solution [7]. The relationship between the sorbed concentration and the concentration remaining in solution at equilibrium is referred to as the sorption isotherm because the experiments are performed at constant temperature. The simplest expression of equilibrium sorption is the linear sorption isotherm. The rate of sorption is described by distribution coefficient $\mathrm{K}_{\mathrm{d}}$, which is defined as the ratio of the sorbed contaminant concentration to the dissolved contaminant concentration. For systems described by a linear isotherm, $\mathrm{K}_{\mathrm{d}}$ is a constant [7].

The non-sorbing elements C, Cl, Se are for many national concepts (Belgian, Swedish, Swiss) characterized by zero value. The distribution coefficient of iodine is zero in Swedish and Belgian model and has very low range of values in Swiss concept [8].

Distribution coefficients are in the model described by discrete distribution $(P=0.7$ for the reference value, with $P=0.15$ for the optimistic and pessimistic values) which was selected to give sufficient weight to the reference value, while acknowledging that the limiting values have a significant likelihood [4].

\subsubsection{Solubility}

The concentration of the contaminant can be limited by its solubility. The solubility of the element depends on several factors such as the groundwater composition, temperature, pressure, etc. If the amount of the element is greater than its solubility limit, precipitations are formed. A solubility limit refers to an individual element (not nuclides). This means that the solubility of the element is divided between its isotopes based on the isotopic ratio. Before the precipitation occurs, the nuclide must reach a saturation concentration values in a transport medium.

Only the radionuclides without any solubility limit (C, Cl, I, Cs) are the same for many national concepts and therefore are not evaluated by any uncertainty range or distribution (Swedish, Swiss, Belgian). There are of course radionuclides whose solubility values differ in individual national concepts. Solubility limits for many nuclides varies several orders of magnitude between the individual national concepts [8].

Solubility limits are in the model described by discrete distribution with the same probability for the reference, optimistic and pessimistic values like in the case of distribution coefficients [4]. 


\subsection{Results on Probabilistic Simulations}

To take into account uncertainties of solubility limits and distribution coefficients, the probability analysis was performed.

Following graphical outputs, which are provided by the simulation tool Gold Sim, illustrate the impact of uncertainties of selected parameters on the release rates of radionuclides from the near field to the surrounding host rock. These figures show the ranges of release rates which are caused by specifying the input parameters as probability distributions (discrete distribution in both cases). The ranges are bounded by maximum and minimum values and a black curve in these figures represents the median of all realizations. This method of evaluation allows to identify the time intervals at which due to the uncertainty in the calculation of the changing parameters there is the greatest variance in the release rate. Figure $\mathbf{3}$ and Figure $\mathbf{4}$ represent the influence of solubility uncertainty of ${ }^{79} \mathrm{Se}$ and ${ }^{99} \mathrm{Tcon}$ the release rates from the EBS. Significant time-dependent variance in the release rate of ${ }^{79} \mathrm{Se}$ is caused by much greater variance between the upper and lower cut value (pessimistic and optimistic) in the discrete distribution of the solubility of ${ }^{79}$ Sethan in the distribution of the solubility of ${ }^{99} \mathrm{Tc}$.

Figure 5 and Figure 6 illustrate the impact of solubility and distribution coefficient uncertainties on the total release rates from the EBS. It can be seen that the variance of the total release rate in the case of solubility uncertainty is almost two orders of magnitude within a long period of time.

\section{Conclusions}

Based on the analysis of the results many of actinides (e.g. ${ }^{233} \mathrm{U},{ }^{236} \mathrm{U}{ }^{232} \mathrm{Th}$ ) are effectively sorbed on the buffer material and, therefore, migrate slowly through the bentonite buffer. The release rates of radionuclides which concentration is controlled by solubility (e. g. ${ }^{227} \mathrm{Ac},{ }^{210} \mathrm{~Pb},{ }^{226} \mathrm{Ra}$ ) are reduced in relation to their inventories and half-lives.

In Slovakia, there has not been selected a final locality for a geological disposal facility hence the assessment of long-term safety was performed for a hypothetical geological repository in a hypothetical rock environment. To evaluate the impact of uncertainties related to this fact, the probabilistic sensitivity analysis of parameters

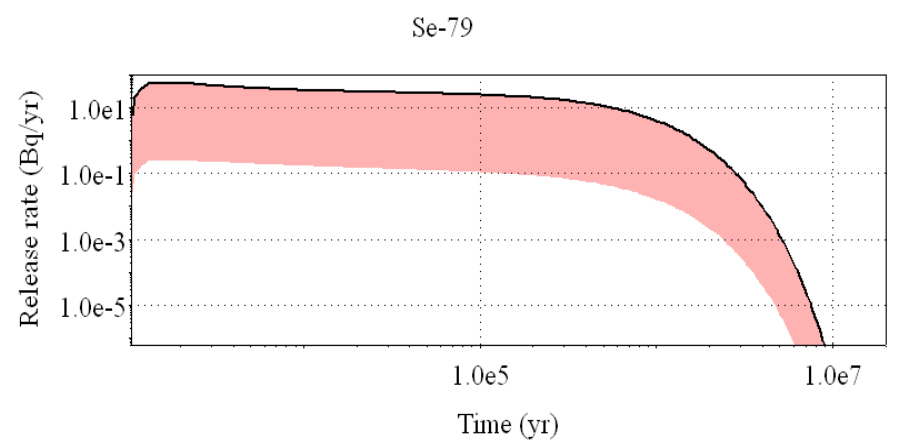

Figure 3. The impact of solubility uncertainty of ${ }^{79}$ Se on the release rate of this nuclide from the EBS.

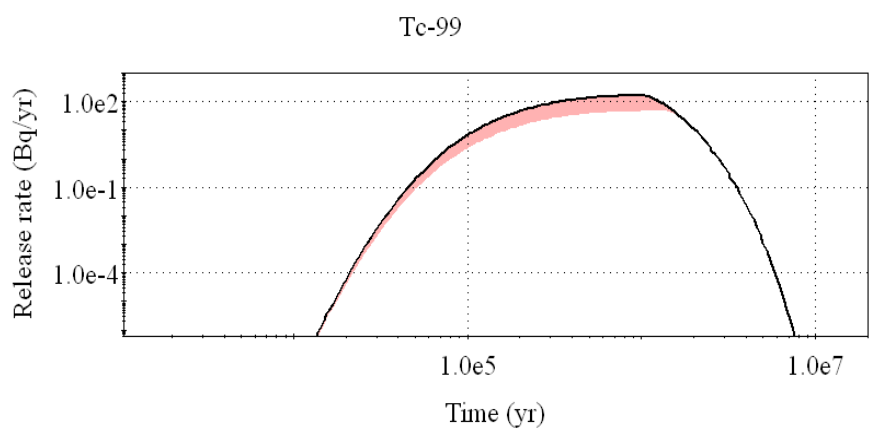

Figure 4. The impact of solubility uncertainty of ${ }^{99} \mathrm{Tc}$ on the release rate of this nuclide from the EBS. 
All nuclides

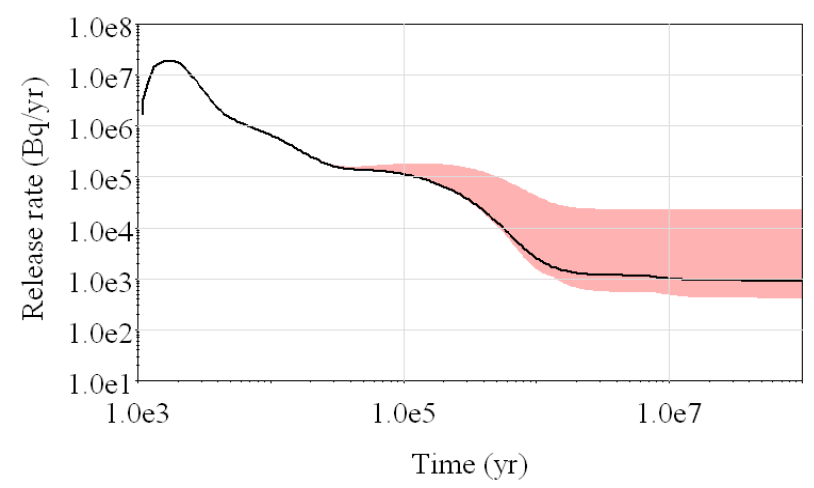

Figure 5. The impact of solubility uncertainty of all nuclides on the total release rate from the EBS.

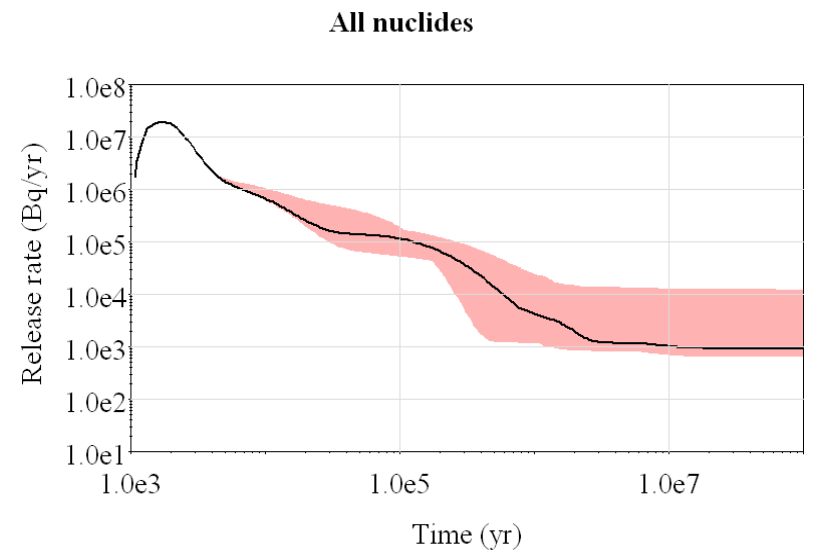

Figure 6. The impact of distribution coefficient uncertainty of all nuclides on the total release rate from the EBS.

connected with the solubility and sorption in the near field was performed. The chosen method of evaluation allows to identify the time intervals at which due to the uncertainty in the calculation of the changing parameters there is the greatest variance in the release rate.

\section{Acknowledgements}

This project has been supported by the Slovak Grant Agency for Science through grant VEGA 1/0796/13.

\section{References}

[1] National Nuclear Fund for Decommissioning of the Nuclear Installations and for Handling of Spent Fuel and Radioactive Waste (2014) The Strategy for the Final Stage of Peaceful Utilization of the Nuclear Energy in SR. (In Slovak)

[2] Nuclear and Decommissioning Company (2014) Interim Spent Fuel Storage. http://www.javys.sk/en/nuclear-facilities/interim-spent-fuel-storage/interim-spent-fuel-storage

[3] Poinssot, C., Ferry, C., Kelm, M., et al. (2004) Spent Fuel Stability under Repository Conditions-Final Report of the European Project. European Commission, November 2001-October 2004. 104 s. CONTRACT No. FIKW-CT-200100192 SFS.

[4] Project Opalinus Clay (2002) Demonstration of Disposal Feasibility for Spent Fuel, Vitrified High-Level Waste and Long-Lived Intermediate-Level Waste (Entsorgungsnachweis). Switzerland: Nagra, 2002. Nagra Technical Report ISSN 1015-2636.

[5] Radioactive Waste Repository Authority (1999) Reference Project of Geological Repository: Technological Part. Czech Republic: RAWRA. 
[6] GOLDSIM Technology Group LLC (2010) GoldSim Contaminant Transport Module, User`s Guide. Washington, USA.

[7] United States Environmental Protection Agency (2015) Important Processes Affecting the Fate and Transport of Organic Compounds in the Subsurface. http://www.epa.gov/superfund/health/conmedia/gwdocs/pdfs/protoapb.pdf

[8] Prváková, S. and Nilsson, K.F. (2006) Treatment of Data Uncertainty for the Modelling of Radionuclide Migration in Geological Repository. Office for Official Publications of the European Communities, Luxembourg, 44 p. 